\title{
Boundary Layer Flow of Ferrofluid over a Stretching Sheet in the presence of Heat Source/Sink
}

\author{
Annamma Abraham* and L. S. Rani Titus ${ }^{\dagger}$
}

\section{Abstract}

The boundary layer flow of ferrofluid over a stretching sheet with heat source is considered. It is assumed that the magnetic field is sufficiently strong enough to saturate the ferrofluid and the variation of magnetization with temperature can be approximated by a linear function of temperature difference. The boundary layer approximation is used and the transformed governing differential equations are solved using the Shooting technique based on Runge - Kutta Fehlberg and Newton Raphson methods. The effects of various parameters on velocity profiles and wall heat transfer are presented graphically. The results have possible industrial applications in liquid based systems involving stretchable materials.

Key words: Ferrofluid, magnetic dipole, stretching sheet, heat source, sink, Prandtl number, shooting method.

\footnotetext{
* BMS Institute of Technology, Bangalore

† Jyoti Nivas Degree College (Autonomous), Bangalore
} 


\section{Introduction}

An interesting fluid mechanical application is found in polymer extrusion processes, where the object on passing between two closely placed solid blocks is stretched into a liquid region. The stretching imparts a unidirectional orientation to the extradite, there by improving its mechanical properties [1].

Ferrofluids are artificially synthesized and consist of highly concentrated colloidal suspensions of fine magnetic particles in a non-conducting carrier fluid. The resulting fluid behaves like a normal fluid except that it experiences a force due to the magnetization. Further, the magnetization of the ferrofluid depends upon the temperature. This thermo magnetic coupling makes ferrofluids useful in various practical applications, see e.g. , [2] and [3]. Siddheshwar and Abraham [4,5] studied the Effects of boundary temperatures/body force on Rayleigh-Benard convection in a ferromagnetic fluid.

The two classical problems in fluid mechanics, namely the Blasius boundary layer flow along a flat plate and the stagnation point flow were extended for a saturated ferrofluid under the combined influence of thermal and magnetic field gradients by Neuringer [6]. The flow of a viscous fluid past a linearly stretching surface in otherwise quiescent surroundings was first considered by Crane [7] for a Newtonian fluid and subsequently extended to fluids obeying non-Newtonian constitutive equations. Andersson and Valnes [8] extended Crane's problem by studying the influence of the magnetic field, due to a magnetic dipole, on a shear driven motion (flow over a stretching sheet) of a viscous non-conducting Ferrofluid. The fluid flow was formulated as a five parameter problem and the influence of the magneto-thermomechanical coupling was explored numerically. It was concluded that the primary effect of the magnetic field was to decelerate the fluid motion as compared to the hydrodynamic case.

The difficulties encountered in using shooting technique are mentioned in [9], such as boundary value problems involving infinity, its sensitivity to the choice of the missing initial conditions and the inherent instability. In Abel et al [10] detailed explanation 
on not only the shooting technique applied to boundary value problems involving a boundary at infinity but also a scientific method of choosing initial values is given.

Siddheshwar and Mahabaleswar [11] studied the effects of radiation and heat source on MHD flow of a viscoelastic liquid and the governing differential equations were solved analytically. Studies on the flow of viscoelastic fluid over a stretching sheet were also reported by Anderson [12], Abel et al. [13, 14] and Arnold et al. [15]

In this paper the influence of the magnetic field due to magnetic dipole on the shear driven motion of a viscous and nonconducting ferrofluid due to stretching is explored. The focus of attention shall be on the magneto thermo-mechanical interaction and heat transfer at the stretching sheet.

\section{Mathematical formulation of the problem}

A steady two-dimensional flow of an incompressible, viscous and electrically non-conducting ferrofluid driven by an impermeable

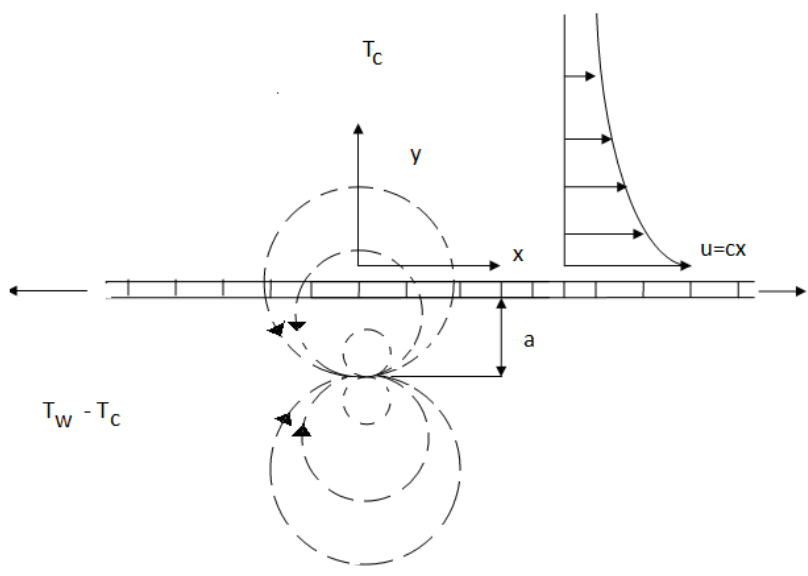

Figure 1: Schematic representation of flow configuration.

The broken lines represent the magnetic field

stretching sheet is considered. The flow is caused by the action of two equal and opposite forces along the horizontal direction which 
is taken as the $x$-axis, and the direction normal to the flow as the yaxis. The sheet is stretched with a velocity $u(x)$ which is proportional to the distance from the origin. A magnetic dipole is located some distance below the sheet. The centre of the dipole lies on the $y$-axis at a distance ' $a$ ' below the $x$-axis. Its magnetic field points in the positive $x$-direction and the strength of the magnetic field is sufficient to saturate the ferrofluid. The stretching sheet is kept at a fixed temperature $T_{w}$ below the Curie temperature $T_{\mathcal{c}}$, while the fluid elements far away from the sheet are assumed to be at temperature $T=T_{c}$ and hence incapable of being magnetized until they begin to cool upon entering the thermal boundary layer adjacent to the sheet.

The equations governing the flow and heat transfer in a ferrofluid are as follows:

$$
\begin{gathered}
\frac{\partial u}{\partial x}+\frac{\partial v}{\partial y}=0 \\
u \frac{\partial u}{\partial x}+v \frac{\partial v}{\partial y}=\frac{\mu}{\rho} \frac{\partial^{2} u}{\partial y^{2}}+\frac{\mu_{0}}{\rho} M \frac{\partial H}{\partial x} \\
\rho C_{p}\left(u \frac{\partial T}{\partial x}+v \frac{\partial T}{\partial y}\right)+\mu_{0} T \frac{\partial M}{\partial T}\left(u \frac{\partial H}{\partial x}+v \frac{\partial H}{\partial y}\right)=k \frac{\partial^{2} T}{\partial y^{2}}+Q\left(T_{c}-T\right)
\end{gathered}
$$

where $u$ and $v$ are the velocity components along $x$ and $y$ directions respectively, $\rho$ is the fluid density, $\mu$ the dynamic viscosity, $v=\frac{\mu}{\rho}$ the kinematic viscosity, $C_{p}$ specific heat at constant pressure, $k$ the thermal conductivity, $\mu_{0}$ the magnetic permeability, $M$ the magnetization, $H$ the magnetic field, $T$ the temperature of the fluid and $Q$ the heat source (sink).

The assumed boundary conditions for solving the above equations are

$$
\begin{aligned}
& u=c x, v=0, T=T_{w} \text { at } y=0 \\
& u \rightarrow 0, T=T_{c} \text { as } y \rightarrow \infty
\end{aligned}
$$


The flow of ferrofluid is affected by the magnetic field due to the magnetic dipole whose magnetic scalar potential is given by

$$
\phi=\frac{\alpha^{\prime}}{2 \pi}\left(\frac{x}{x^{2}+(y+a)^{2}}\right),
$$

where $\alpha^{\prime}$ is the dipole moment per unit length.

The magnetic field $H$ has the components

$$
\begin{aligned}
& H_{x}=-\frac{\partial \phi}{\partial x}=\frac{\alpha^{\prime}}{2 \pi} \frac{x^{2}-(y+a)^{2}}{\left(x^{2}+(y+a)^{2}\right)^{2}}, \\
& H_{y}=-\frac{\partial \phi}{\partial y}=\frac{\alpha^{\prime}}{2 \pi} \frac{2 x(y+a)}{\left(x^{2}+(y+a)^{2}\right)^{2}}
\end{aligned}
$$

Since the magnetic body force is proportional to the gradient of the magnitude of $H$, we obtain from $H=\sqrt{\left(\frac{\partial \phi}{\partial x}\right)^{2}+\left(\frac{\partial \phi}{\partial y}\right)^{2}}$ that

$$
\frac{\partial H}{\partial x}=-\frac{\alpha^{\prime}}{2 \pi}\left(\frac{2 x}{(y+a)^{4}}\right), \quad \frac{\partial H}{\partial y}=\frac{\alpha^{\prime}}{2 \pi}\left(\frac{-2}{(y+a)^{3}}+\frac{4 x^{2}}{(y+a)^{5}}\right)
$$

Variation of magnetization $M$ with temperature $T$ is approximated by a linear equation $\quad M=K\left(T_{c}-T\right)$

where $K$ is the pyromagnetic coefficient

\section{Solution Procedure}

We shall now introduce the non - dimensional variables

$$
\begin{aligned}
& \xi=\sqrt{\frac{c}{v}} x, \eta=\sqrt{\frac{c}{v}} y, U=\sqrt{\frac{1}{c v}} u, V=\sqrt{\frac{1}{c v}} v \\
& \theta(\xi, \eta)=\frac{T_{c}-T}{T_{c}-T_{w}}=\theta_{1}(\eta)+\xi^{2} \theta_{2}(\eta)
\end{aligned}
$$


In order to convert the partial differential equation (1), (2) , (3) and (4) into ordinary differential equation the following similarity transformation is used.

$$
\Psi(\xi, \eta)=\xi f(\eta)
$$

The velocity components $\mathrm{U}$ and $\mathrm{V}$ are related according to

$$
\begin{gathered}
U=\frac{\partial \Psi}{\partial \eta}=\xi f^{\prime}(\eta), \\
\mathrm{V}=-\frac{\partial \Psi}{\partial \xi}=-f(\eta),
\end{gathered}
$$

where the prime denotes differentiation with respect to $\eta$ and

We obtain the following boundary value problem

$$
\begin{gathered}
f^{\prime \prime \prime}+f f^{\prime \prime}-\left(f^{\prime}\right)^{2}-\frac{2 \beta \theta_{1}}{(\eta+\alpha)^{4}}=0 \\
\theta_{1}^{\prime \prime}+\operatorname{Pr}\left(f \theta_{1}^{\prime}-Q_{s} \theta_{1}\right)+\frac{2 \beta f \lambda}{(\eta+\alpha)^{3}}\left(\theta_{1}-\varepsilon\right)=0 \\
\theta_{2}^{\prime \prime}-\operatorname{Pr}\left(2 f^{\prime} \theta_{2}-f \theta_{2}^{\prime}+Q_{s} \theta_{2}\right)+\frac{2 \lambda \beta f \theta_{2}}{(\eta+\alpha)^{3}}-\lambda \beta\left(\theta_{1}-\varepsilon\right)\left[\frac{2 f^{\prime}}{(\eta+\alpha)^{4}}+\frac{4 f}{(\eta+\alpha)^{5}}\right]=0 \\
f(0)=0, f^{\prime}(0)=1, \theta_{1}(0)=1, \theta_{2}(0)=0 \text { at } \eta=0 \\
f^{\prime} \rightarrow 0, \theta_{1} \rightarrow 0, \theta_{2} \rightarrow 0 \text { as } \eta \rightarrow \infty
\end{gathered}
$$

The six dimensionless parameters, which appear explicitly in the transformed problem, are the Prandtl number $\mathrm{Pr}$, the viscous dissipation parameter $\lambda$, the dimensionless Curie temperature $\varepsilon$, the ferrohydrodynamic interaction parameter $\beta$, the heat source/sink parameter $\mathrm{Q}_{\mathrm{s}}$ and the dimensionless distance $\alpha$ from the origin to the center of the magnetic pole, defined respectively as 


$$
\begin{aligned}
& \operatorname{Pr}=\frac{\mu C_{p}}{k}, \\
& \lambda=\frac{c \mu^{2}}{\rho k\left(T_{c}-T_{w}\right)}, \\
& \varepsilon=\frac{T_{c}}{T_{c}-T_{w}}, \\
& \beta=\frac{\alpha^{\prime} \rho}{2 \pi \mu^{2}} \mu_{0} K\left(T_{c}-T_{w}\right) \\
& Q_{s}=\frac{Q}{c \rho C_{p}} \\
& \alpha=\left(\frac{c \rho a^{2}}{\mu}\right)^{\frac{1}{2}} .
\end{aligned}
$$

The local skin friction coefficient $C_{f}$, which is a dimensionless form of the shear stress $\tau$ at the sheet is given by

$$
C_{f}=\frac{-2 \tau_{x y}}{\rho(c x)^{2}}=-2 f^{\prime \prime}(0) \operatorname{Re}_{x}^{-\frac{1}{2}}
$$

and the local heat flux as the local Nusselt number is as follows

$$
N u_{x}=-\operatorname{Re}_{x}^{\frac{1}{2}}\left[\theta_{1}^{\prime}(0)+\xi^{2} \theta_{2}^{\prime}(0)\right]
$$

The three coupled differential equations (15) to (17) subject to the boundary conditions (18) and (19) constitute a non - linear two point boundary value problem, which is solved by means of a standard shooting technique. The higher order ODEs are formulated as first - order equations and the resulting set of seven first - order equations can be integrated as an initial value problem using the adaptive stepping Runge - Kutta - Fehlberg method. Trial 
values of $f^{\prime \prime}(0), \theta_{1}^{\prime}(0), \theta_{2}^{\prime}(0)$ were adjusted iteratively by Newton Raphson's method to assure a quadratic convergence of the iterations required in order to fulfill the outer boundary conditions (19).

\section{Results and discussion}

The magnetic effects on the flow are indicated by the single parameter $\beta$. When $\beta=0$ the magnetic effects disappear and the momentum and energy equations decouple. The effects of $\beta$ on velocity and temperature when the heat source/sink parameter $Q_{s}=-0.5$ are shown in figure 2(a). The influence of $\beta$ on the velocity
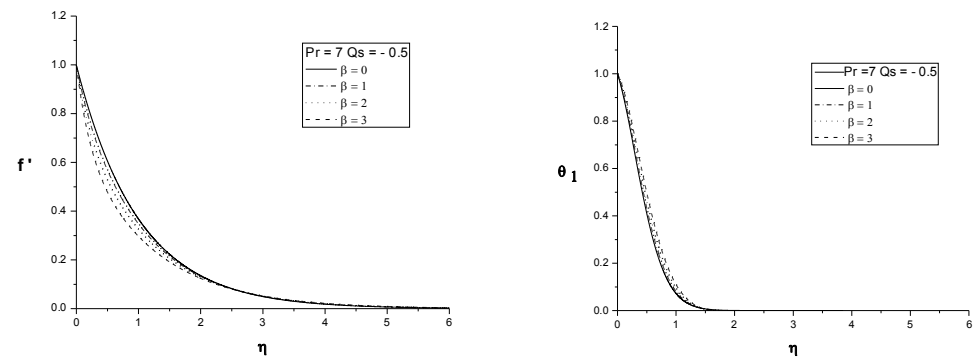

Figure 2: Effect of Ferrohydrodynamic interaction parameter $\beta$ on Velocity and temperature profile

field is the reduction in $\mathrm{f}^{\prime}$ for increasing $\beta$. This transverse contraction of the velocity boundary layer is due to the applied magnetic field, which counters the motion. Hence $\beta$ has a regulating effect on the fluid as it regulates the velocity of the flow. The increased skin friction due to the effect of $\beta$ on the velocity profile enhances the heat transfer. Thus the effect of $\beta$ on the temperature profile is the increase in $\theta_{1}$ for increasing $\beta$ as shown in figure 2(b). 


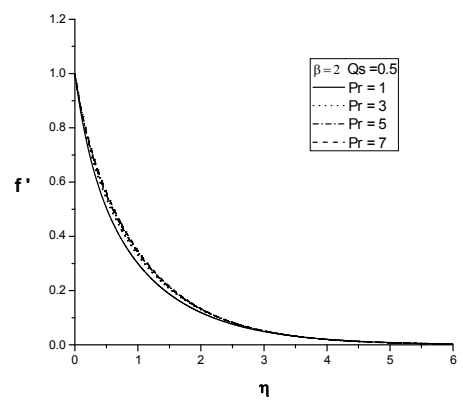

Figure 3(a) and 3(b) illustrate the effect of the Prandtl number Pr on velocity and temperature profiles respectively. When Pr number is large the velocity boundary layer is thick compared to the temperature boundary which decreases the magnitude of $\theta(\eta)$, thus reducing the heat transfer. From figure 4 the effect of internal heat generation (source/sink) is to dampen or enhance the heat transport in a linear fashion.
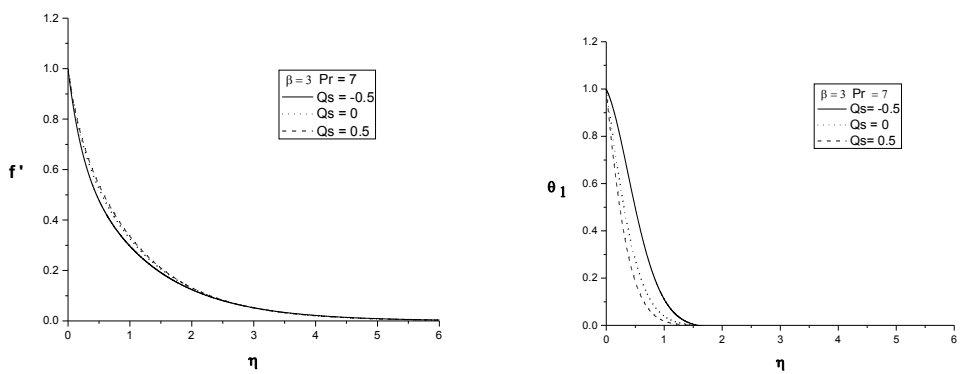

Figure 4: Effect of $\mathrm{Q}_{\mathrm{s}}$ on Velocity and temperature profiles

In the presence of the heat sink the decrease in the velocity profile is more for the increasing values of $\beta$ as compared to that in the presence/absence of heat source. Thus Qs enhances or dampens the effect of $\beta$ depending on whether Qs is a sink or a source. Consequently the variation in temperature is more pronounced for the increase in $\beta$ in case of sink as compared to source. 
Hence it can be concluded that the presence of heat source (sink) controls the effect of the magneto-thermomechanical interaction which decelerates the flow along the stretching sheet thereby influencing the heat transfer rate.

\section{Acknowledgement}

The authors would like to acknowledge their respective institutions for their support.

\section{References}

[1] E. G. Fisher, 1976, Extrusion of Plastics, Third Ed., London, Newnes-Butterworld.

[2] J. L. Neuringer, R. E. Rosensweig, 1964, Ferrohydrodynamics. Phys. Fluids, 7, 1927 - 1937.

[3] R. L. Bailey, 1983, Lesser known applications of ferrofluids. J. Magnetism Magn. Mater. , 39, 178 - 182.

[4] P.G Siddheshwar, A. Abraham, 2003, Effect of time- periodic boundary temperatures/ body force on Rayleigh-Benard convection in a ferromagnetic fluid, Acta Mechanica, 161, 131-150.

[5] Annamma Abraham, 2001, Convective instability in magnetic fluids and polarised dielectric liquids, Ph.D. Thesis, Bangalore University.

[6] J. L. Neuringer, 1966, Some viscous flows of a saturated ferrofluid under the combined influence of thermal and magnetic field gradients, Int. J. Non - linear Mech. , 1, 123 127.

[7] L. J. Crane, 1970, Flow past a stretching plate, J. Appl. Math. Phys. (ZAMP) 21, 645- 647.

[8] H. I. Andersson and O.A. Valnes, 1998, Flow of a heated Ferrofluid over a stretching sheet in the presence of a magnetic dipole, Acta Mechanica , 128, $39-47$. 
[9] E.E. Tzirtzilakis, N. G. Kafoussias, 2003, Biomagnetic fluid flow over a stretching sheet with non - Linear Temperature dependant magnetization, ZAMP , 54, $551-565$.

[10] M.S. Abel, P.G. Siddheshwar, N. Mahesha, 2011, Numerical solution of the momentum and Heat transfer equations for a hydromagnetic flow due to a stretching sheet of a non uniform property micro polar liquid, Applied Mathematics and computation, 217, 5895-5909.

[11] P.G.Siddheshwar,U.S.Mahabaleshwar, 2005, Effect of radiation and heat source on MHD flow of a viscoelastic liquid and heat transfer over a stretching sheet, Int. J. NonLinearMech.40, 807-820.

[12] H.I.Andersson, 1992, MHD flow of a viscoelastic fluid past a stretching surface, Acta Mech., 95, 227-230.

[13] M. Subhas Abel, Mahantesh M., Nandeppanavar, 2009, Heat transfer in MHD viscoelastic boundary Layer flow over a stretching sheet with non-uniform heat source/sink, Communications in Nonlinear Science and Numerical Simulation, 14, 5, 2120-2131.

[14] M. Subhas Abel, N. Mahesha, 2008, Heat transfer in MHD viscoelastic fluid flow over stretching sheet with variable thermal conductivity, non-uniform heat source and radiation, Applied Mathematical Modelling, 32, 1965-1983.

[15] J. Arnold, A. Asir, S. Somasundaram, T. Christopher, 2010, Heat transfer in a viscoelastic boundary layer flow over a stretching sheet, Int. J. of Heat and Mass Transfer, 53, (5), 1112-1118. 